\title{
Hertz-To-Terahertz Dielectric Response of Nanoconfined Water Molecules ${ }^{\dagger}$
}

\author{
Boris Gorshunov 1,*, Mikhail Belyanchikov ${ }^{1}$, Maxim Savinov ${ }^{2}$, Peter Bednyakov ${ }^{2}$, \\ Zakhar Bedran 1, Viktor Thomas 3, Viktor Torgashev 4, Vladimir Anzin 1, Alois Loidl 5, \\ Peter Lunkenheimer ${ }^{5}$, Elena Zhukova ${ }^{1}$, Ece Uykur ${ }^{6}$ and Martin Dressel 6 \\ 1 Moscow Institute of Physics and Technology, Dolgoprudny, 141700 Moscow, Russia \\ 2 Institute of Physics AS CR, Na Slovance 2, 18221 Praha 8, Czech Republic \\ 3 Institute of Geology and Mineralogy, RAS, 630090 Novosibirsk, Russia \\ 4 Faculty of Physics, Southern Federal University, 344090 Rostov-on-Don, Russia \\ 5 Experimental Physics V, University of Augsburg, 86135 Augsburg, Germany \\ 6 Physikalisches Institut, Universität Stuttgart, 70569 Stuttgart, Germany \\ * Correspondence: bpgorshunov@gmail.com \\ + Presented at the 37th International Symposium on Dynamical Properties of Solids - DyProSo 2019, Ferrara, \\ Italy, 8-12 September 2019.
}

Published: 5 September 2019

Recently, considerable attention has been given to the properties of systems that contain interacting point electric dipoles. Such systems are expected to manifest rich variety of exotic phases resulting from competition between the dipole-dipole coupling and disordering effects, both dependent of various factors, like spatial symmetry and dimensionality of the dipoles locations, presence of defects and impurities, frustration, etc. An additional issue concerns the study of the dynamics of dipoles as interacting quantum rotors that experience a multi-well localizing potential in presence of hindering effects and quantum rotational tunneling. It is important, that the electrostatic coupling among the dipoles makes such systems qualitatively different from their magnetic counterparts. Since interacting spins have been thoroughly studied during last decades, significant progress has been achieved in understanding the underlying magnetic physics. In electric dipolar systems, the interplay of quantum tunneling, fluctuations and frustration provides with the possibility to realize new exotic phases, like (anti)ferroelectricity, quantum electric dipolar liquids and glasses, lead to quantum critical phenomena and quantum phase transitions. Understanding the nature of the corresponding phases, their formation and relations with magnetic counterparts is of great fundamental and technological interest, but is presently at its infancy.

Prospective playground for corresponding studies is provided by dielectrics whose crystal lattice contains voids filled with electric-polar molecules that only weakly interact with surrounding ions and are thus nearly free, but "feel" each other via long-range electric dipole-dipole interaction. Corresponding broad-band spectroscopic studies of the gemstone beryl with $0.5 \mathrm{~nm}$ sized pores hosting polar $\mathrm{H}_{2} \mathrm{O}$ molecules (each carrying a dipole moment of $\approx 1.85$ Debye) allowed to discover incipient ferroelectricity within the $\mathrm{H}_{2} \mathrm{O}$ dipoles, along with a rich set of single-particle excitations at terahertz-infrared frequencies [1-5]. It was suggested that the (anti)ferroelectric phase transition into the macroscopically ordered state was suppressed by quantum tunnelling of $\mathrm{H}_{2} \mathrm{O}$ molecules between $1 \mathrm{meV}$ deep wells of the localizing six-fold potential. Here, we present detailed spectroscopic studies of single-particle and collective vibrational states of a network of $\mathrm{H}_{2} \mathrm{O}$ molecules hosted by orthorhombic crystal lattice of cordierite. Unlike hexagonal beryl, water molecules in cordierite experience 2-well localizing potential while rotating $360^{\circ}$ around the c-axis, with the wells separated by an order of magnitude higher energy barriers of $\approx 10 \mathrm{meV}$.

Using terahertz $(\mathrm{THz})$, radio-frequency $(\mathrm{RF})$ and microwave $(\mu \mathrm{W})$ spectroscopies, we measure polarization-dependent (E-field component of the probing radiation parallel to $a, b$ and 
c axes) spectra of the complex dielectric permittivity $\varepsilon(v)=\varepsilon^{\prime}(v)+i \varepsilon^{\prime \prime}(v)$ and AC conductivity $\sigma(v)=$ $\sigma 1(v)+i \sigma 2(v)$ of hydrous cordierite in the range $v=1 \mathrm{~Hz}-3 \mathrm{THz}$ and at temperatures $0.3 \mathrm{~K}-300 \mathrm{~K}$. Measurements on water-free (annealed in vacuum) crystals allowed us to extract the spectra determined exclusively by water molecules. In the $\mathrm{THz}$ range, $0.3 \mathrm{THz}-3 \mathrm{THz}$, rich sets of highly anisotropic soft excitations are discovered. Applying density-functional and molecular dynamics analyses allowed us to associate the origin of the excitations with complex librational-rotational vibrations of the nanoconfined polar water molecules. In the RF- $\mu \mathrm{W}$ ranges, $1 \mathrm{~Hz}-1 \mathrm{GHz}$, for the E I l a polarization we discover a strongly temperature-dependent overdamped excitation and assign its origin to relaxational dynamics of separate molecular dipoles within the confining cage. Below $3 \mathrm{~K}$, we detect weak signatures of a phase transition into a glassy state formed by frozen water dipoles. We will perform comparative analysis of the results obtained on water dipoles arranged in hexagonal (beryl matrix) and in orthorhombic (cordierite matrix) arrays.

Funding: Authors acknowledge support from RFBR grants 18-32-00286 and 18-32-20186, DFG via DR228/61-1 and Center of Integrated Quantum Science and Technology IQST Stuttgart/Ulm.

\section{References}

1. Gorshunov, B.P.; Zhukova, E.S.; Torgashev, V.I.; Lebedev, V.V.; Shakurov, G.M.S.; Kremer, R.K.; Pestrjakov, E.V.; Thomas, V.G.; Fursenko, D.A.; Dressel, M. Quantum behavior of water molecules confined to nanocavities in gemstones. J. Phys. Chem. Lett. 2013, 4, 2015-2020.

2. Gorshunov, B.P.; Torgashev, V.I.; Zhukova, E.S.; Thomas, V.G.; Belyanchikov, M.A.; Kadlec, C.; Kadlec, F.; Savinov, M.; Ostapchuk, T.; Petzelt, J.; et al. Incipient ferroelectricity of water molecules confined to nano-channels of beryl. Nat. Commun. 2016, 7, 12842.

3. Belyanchikov, M.A.; Zhukova, E.S.; Tretiak, S.; Zhugayevych, A.; Dressel, M.; Uhlig, F.; Smiatek, J.; Fyta, M.; Thomas, V.G.; Gorshunov, B.P. Vibrational states of nano-confined water molecules in beryl investigated by first-principles calculations and optical experiments. Phys. Chem. Chem. Phys. 2017, 19, 30740-30748.

4. Zhukova, E.S.; Belyanchikov M.A.; Savinov M.; Bednyakov P.; Thomas V.G.; Kadyrov L.S.; Simchuk E.A.; Bedran Z.V.; Torgashev V.I; Dudka A.; et al. $\mathrm{H}_{2} \mathrm{O}$ Molecules Hosted By a Crystalline Matrix-New State of Water? EPJ Web Conf. 2018, 195, 06018.

5. Dressel, M.; Zhukova E.S.; Thomas V.G.; Gorshunov B.P. Quantum Electric Dipole Lattice of Water Molecules Confined to Nanocavities in Beryl. J. Infrared Millime. Terahertz Waves 2018, 39, 799.

(C) 2019 by the authors. Licensee MDPI, Basel, Switzerland. This article is an open access article distributed under the terms and conditions of the Creative Commons Attribution (CC BY) license (http://creativecommons.org/licenses/by/4.0/). 